\title{
THE RELATIVE INTENSITIES OF THE CORONAL AND OTHER FORBIDDEN LINES
}

\author{
I. S. Bowen and P. Swings \\ Mount Wilson Observatory \\ Received September 18, 1946
}

\section{ABSTRACT}

The behavior of the coronal lines in RS Ophiuchi, T Pyxidis, and T Coronae Borealis is in accord with the physical properties of these lines as predicted from Edlén's identifications. Observed anomalies in the relative abundance of certain ions in these and similar objects may be explained by irregularities in the intensity-curve of the primary exciting radiation of the central star, caused by $H$ and $H e$ II continuous absorption. One line in the RS Oph spectrum is identified with [ $K \mathrm{XI}]$.

In 1941, Bengt Edlén identified the coronal lines as forbidden lines of $A, C a, F e$, and $N i$ in the ninth to fifteenth stages of ionization. Strong evidence, from the physical side, for these identifications was provided by Edlén's own measurements of the extreme ultraviolet spectra of these ions.

From the astrophysical standpoint the presence of these extremely high stages of ionization in the neighborhood of the sun raised many difficult questions. Particularly disturbing was the presence of very highly ionized ions in the corona along with neutral and singly ionized atoms in the chromosphere and prominences, while all intermediate stages of ionization seemed to be absent.

The two strongest coronal lines, $\lambda 5303$ and $\lambda 6374$, have been observed in the three stars, RS Ophiuchi, ${ }^{1} \mathrm{~T}$ Pyxidis, ${ }^{2}$ and $\mathrm{T}$ Coronae Borealis $;{ }^{3}$ three fainter coronal lines also have been detected in RS Oph. It becomes of interest, therefore, to consider whether the behavior of the coronal lines in these objects is in accord with the properties of the ions concerned, as predicted by physical theory. In particular, it is important to note whether this behavior throws any light on the difficulty already mentioned.

Table 1 lists all the coronal lines that have been definitely observed in at least one object besides the corona itself. The third column gives the transition probability $A_{\mathrm{m}}$ of each line; the fourth to seventh columns show the observed intensities in the various objects. For comparison, the transition probabilities and the intensities of the strongest lines of $[\mathrm{Fe}$ vII] and $[\mathrm{O} \mathrm{III}]$ also are listed.

In passing from the $F e \mathrm{x}$ of the coronal spectra to the lower stages of ionization of $F e$, the first stage having forbidden transitions in the observable range is $F e$ viI. Aside from the difference in ionization, the chief difference between the lines of [ $F e$ VII] and the strongest coronal lines assigned to $[F e \mathrm{x}]$ and $[F e \mathrm{XIV}]$ lies in the fact that the transition probabilities of the coronal lines are greater by a factor of about 100 . One may, therefore, expect a marked decrease in the ratio of the intensities of [ $\mathrm{Fe}$ VII] lines to coronal lines in passing to objects of increasing density. Such a decrease in the relative intensities may be observed if the objects in Table 1 are examined from right to left. That the change in density is a major cause of this shift in the relative intensities is

${ }^{1}$ Observed in 1933 but recently described in more detail by Joy and Swings, $M t$. W. Contr., No. 714; Ap. J., 102, 353, 1945.

${ }^{2}$ A. H. Joy, Pub. A.S.P., 57, 171, 1945.

${ }^{3}$ R. F. Sanford, Pub. A.S.P., 58, 156, 1946; D. B. McLaughlin, Pub. A.S.P., 58, 159, 1946; Herbig and Neubauer, Pub. A.S.P., 58, 196, 1946; J. Dufay, I.A.U. Circular, No. 1036 (Copenhagen, 1946). 
shown by the behavior of the $[O \mathrm{III}]$ lines. In T Pyx the density was evidently very low, since the highly forbidden nebular pair at $\lambda 5007$ and $\lambda 4959$ was nearly as strong as $\lambda 4363$, which has a much higher transition probability. In RS Oph, however, increased density reduced the intensity of the lines of the nebular pair to less than one-tenth the intensity of $\lambda$ 4363. In T CBr, $\lambda 4363$ was the only [O III] line observed, and it appeared only a short time before the disappearance of the coronal lines. No $[O \mathrm{III}]$ lines are observed in the corona. Indeed, with the exception of $\lambda 4363$ in $\mathrm{T} \mathrm{CBr}$ and a few [ $\mathrm{Fe}$ II] lines observed both in $\mathrm{T} \mathrm{CBr}$ and in the chromosphere, ${ }^{4}$ the coronal lines are the only forbidden lines observed either in the sun or in $\mathrm{T} \mathrm{CBr}$ during the period under consideration. Although no direct calculations have been made, there is experimental evidence for believing that the transition probabilities of the $[\mathrm{Fe}$ II] lines are nearly as great as those of the coronal lines. ${ }^{4,5}$

TABIE 1

INTENSITIES OF FORBIDDEN LINES IN VARIOUS SOURCES

\begin{tabular}{|c|c|c|c|c|c|c|}
\hline Ion & $\lambda$ & $\begin{array}{c}A_{m} \\
\left(\operatorname{Sec}^{-1}\right)\end{array}$ & Corona & $\mathrm{TCBr}$ & RS Oph & T Pyx \\
\hline 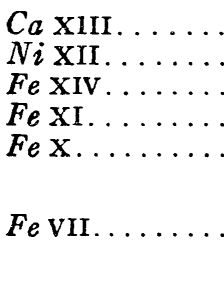 & $\begin{array}{l}4086.3 \\
4231.4 \\
5302.86 \\
3986.9 \\
6374.51 \\
\begin{array}{l}6087.6 \\
5722.6 \\
5163.2 \\
3758.9\end{array}\end{array}$ & $\begin{array}{l}319 \\
237 \\
60 \\
9.5 \\
69 \\
0.49 \\
0.30 \\
0.21 \\
0.37\end{array}$ & $\begin{array}{c}1.0 \\
2.6 \\
100 \\
0.7 \\
8.1\end{array}$ & $\begin{array}{l}\overline{-} \\
\overline{\mathrm{P}^{*}} \\
\overline{\mathrm{P}^{*}} \\
\overline{-} \\
\overline{-} \\
\overline{-}\end{array}$ & $\begin{array}{r}2 \\
4 \\
6 \\
4 \\
20 \\
3 \\
\ldots \\
0 \\
4\end{array}$ & $\begin{array}{c}\cdots \cdots \\
\cdots \\
\cdots \\
1 \\
5 \\
\mathrm{M} \ddagger \\
2 \\
2\end{array}$ \\
\hline$O$ III.......... & $\left\{\begin{array}{l}5006.84 \\
4958.91 \\
4363.21\end{array}\right.$ & $\begin{array}{l}0.016 \\
0.0056 \\
2.8\end{array}$ & & $\bar{t}$ & $\begin{array}{r}3 \\
1 \\
35\end{array}$ & $\begin{array}{l}40 \\
20 \\
50\end{array}$ \\
\hline
\end{tabular}

* Present.

$\dagger$ Absent when the coronal lines first appeared, but present as a narrow line shortly before their disappearance.

$\ddagger$ Masked.

The appearance of the coronal lines in these additional objects therefore provides definite evidence that, as the density varies, the coronal lines behave in just the manner that would be expected from Edlén's identifications. Furthermore, these additional observations give added weight to the suggestion originally made by Joy and Swings ${ }^{1}$ that high density causes the absence of the forbidden lines of the intermediate stages of ionization in certain of these objects.

Other apparent anomalies in the relative abundance of certain ions in these objects may be caused by the operation of the primary mechanism of excitation of the gaseous envelopes. Presumably, the emission spectra of such objects as T CBr, RS Oph, and $T$ Pyx come from a surrounding gaseous cloud excited by a mechanism similar to that which has proved effective in explaining the spectra of the planetary nebulae. ${ }^{6}$ In this mechanism the primary step is the photo-ionization of the gas by the absorption of the continuous ultraviolet radiation of a very hot central star. Since approximately 99 per cent of the atoms in most astronomical objects are either $\mathrm{H}$ or $\mathrm{He}$, these atoms will be the chief absorbers of radiation by this process. Furthermore, in the side of the cloud

${ }^{4}$ Bowen and Menzel, Pub. A.S.P., 40, 332, 1928.

${ }^{5}$ Swings and Struve, $A$ p. $J ., 93,455,1941$.

'I. S. Bowen, $A$ p. J., 81, 1, 1935. 
nearest the hot star this absorption will be limited largely to the ions $\mathrm{H}$ I and $\mathrm{He}$ Ir. The continuous stellar radiation entering the cloud will, therefore, be rapidly depleted in the regions of wave length shortward of the respective limits of absorption of these ions, 912 and $228 \mathrm{~A}$. Throughout most of the cloud the primary exciting radiation will be greatly deficient in these two wave-length ranges. As a result, any ion whose ionization limit falls just short of 912 and $228 \mathrm{~A}$ will not be further ionized as rapidly as ions whose limits fall on either side of these deficient bands in the spectra. This should cause a substantial increase relative to adjacent stages of ionization in the population of ions whose limits fall in these deficient bands.

Because of the deficiency in radiation below $228 \mathrm{~A}$, one would expect an unusual number of atoms in the stages $C$ IV, $N$ IV, $O$ III, $N e$ III, $A$ IV, $K$ IV, $F e$ IV, etc., while the band below 912 A should cause an increase in the abundance of $O \mathrm{I}, S i \mathrm{Ir}$, and $F e$ Ir. One should, therefore, expect low-excitation-potential lines of these ions, either forbidden or permitted, to be abnormally strong where such lines exist in the observable range. Highexcitation-potential lines, usually permitted, are normally formed in the course of recombination of the ion with an electron. This type of line should therefore appear with enhanced intensity for the next lower stage of ionization in each atom.

This effect will be still further enhanced by the presence in the gaseous envelope of strong emission lines of $H$ and $H e$ II in the regions just longward of the respective limits, 912 and $228 \mathrm{~A}$. Thus the absorption of these $H$ and $H e$ II lines will cause an additional increase in the population of the ions whose absorption limits fall just shortward of 912 and $228 \mathrm{~A}$ at the expense of the next lower stage of ionization. For example, the population of $F e$ IV should be still further increased with respect to $F e$ III.

From the wide range of ionization in an object such as RS Oph, it would be inferred that the central star is surrounded by a layer of gas which is relatively thick in terms of the mean free path of the primary exciting radiation. Consequently, this mechanism should be particularly effective in RS Oph in modifying the normal distribution of ions between the various stages. It is, therefore, not surprising to find the lines of permitted $C$ III and $N$ III and forbidden $O$ III, $N e$ III, and $F e$ II abnormally strong relative to the neighboring stages of ionization of these atoms. No forbidden or permitted lines of low excitation potential exist for $F e$ IV in the observable range. However, the transfer of most of the $F e$ in the lower stages of ionization to $F e$ II and $F e$ IV may account for the observed weakness of $[\mathrm{Fe}$ III], [Fe V], and [Fe VI], compared to $[F e \mathrm{II}]$ and $[\mathrm{Fe}$ VII].

The same tendency to favor certain ions has been evident in varying degrees in the spectra of other objects, such as AX Per, CI Cyg, and RX Pup, which show a wide range of stages of ionization. The fact that $[\mathrm{Fe}$ II] and [ $\mathrm{Fe}$ VII] are both strong at the same time, in comparison to the intervening stages, has long been particularly puzzling in these objects. Previously, the interpretation of these effects has been sought in the binary character of the objects or in stratification phenomena. If the present interpretation is correct, it may not be necessary to call on these older explanations.

The behavior of $C a \mathrm{Ir}, F e$ II, $T i$ II, etc., in the hotter stars suggests that the mechanism discussed in this Contribution may play a role in the excitation of the emission lines found in the spectra of many of these objects. Thus in the emission spectra of these stars the lines of $C a \mathrm{II}$, whose series limit at $1044 \mathrm{~A}$ is well outside the $H$ absorption band, are relatively faint compared to the lines of such ions as $F e$ II and $T i$ II. This is in marked contrast with the absorption spectra of stars of early type, in which the $C a$ II lines are normally outstanding among the metallic lines.

Unfortunately, in many objects it is difficult to obtain a definite evaluation of the effectiveness of this mechanism through the determination of the relative populations in the various stages of ionization of a given element. Many of the higher stages of ionization of most elements have no lines of low excitation potential in the observable range. The few available lines of this type are almost invariably forbidden. Furthermore, the transition probabilities of the forbidden lines of successive stages of ionization of ten 
differ in order of magnitude. As discussed on page 1, this causes the relative intensities to be subject to large variations, dependent upon the density in the region of emission. Thus, of the abundant elements, iron has probably the largest number of stages of ionization that are represented with low-excitation-potential lines in the observable range. However, no satisfactory lines are available for $F e$ IV, $F e$ vIII, $F e$ Ix, and $F e$ xII. The transition probabilities of all forbidden lines of $F e$ vI and $F e$ vII are less than $1 \mathrm{sec}^{-1}$. The lines of $[F e \mathrm{x}],[F e \mathrm{XI}],[F e \mathrm{XIII}],[F e \mathrm{XIV}],[F e \mathrm{XV}]$ and probably most of the lines of $[\mathrm{Fe} \mathrm{II}]$ have values in the range from 9 to $100 \mathrm{sec}^{-1}$. The transition probabilities of the $[F e \mathrm{III}],[\mathrm{Fe} \mathrm{v}]$ lines are unknown. Consequently, the $[F e \mathrm{II}]$ lines, like the coronal lines, may in many cases owe to high density part of their enhancement relative to the lines of other stages of ionization. Because of these complications, it is often necessary, therefore, to rely on general trends among the ions of all available atoms for an evaluation of the effectiveness of this mechanism in shifting the distribution between various stages of ionization. In any case, the mechanism is one more factor that must be considered in a quantitative study of the physical conditions in emission-line objects.

In this discussion of the spectrum of RS Oph, one new identification should be noted. The ${ }^{2} \mathrm{P}_{3 / 2}-{ }^{2} \mathrm{P}_{1 / 2}$ transition of $[K \mathrm{XI}]$ was predicted by Edlén at $\lambda 4258.7$ but is not found in the corona. A line of intensity 1 has been observed in RS Oph at exactly this wave length, but it was identified by Joy and Swings as $\lambda 4258.15 \mathrm{Fe}$ II-an identification which is certainly insufficient and probably erroneous. The line $\lambda 4258 \mathrm{Fe}$ II is a component of laboratory intensity 3 in the $b^{4} \mathrm{P}-z^{4} \mathrm{~F}^{0}$ multiplet. The strongest member of this multiplet at $\lambda 4178.85$ (lab. int. 8) is doubtfully attributed, as part of a blend, to a line of intensity 1 in RS Oph. The other components of the multiplet, $\lambda 4296.57$ (lab. int. 6), $\lambda 4122.64$ (lab. int. 4), and $\lambda 4369.40$ (lab.int. 2) are absent in RS Oph. Of the stronger $b^{4} \mathrm{P}-z^{4} D^{\circ}$ multiplet, which has an excitation similar to that of $b^{4} \mathrm{P}-z^{4} \mathrm{~F}^{\circ}$, several components, stronger than $\lambda 4258.15$ in the laboratory (or in $a \mathrm{Cyg}$ ), are not found in RS Oph. It is thus probable that the line observed at $\lambda 4258$ in RS Oph is actually due to $[K \mathrm{xI}]$. The star shows strong $[K \mathrm{IV}]$ and $[K \mathrm{v}]$. 